\title{
IP Based Network Public Address for Intelligent Building Environment
}

\author{
Chui Yew Leong, ${ }^{1}$ Abd Rahman Ramli and ${ }^{2}$ Thinagaran Perumal \\ ${ }^{1}$ Department of Computer and Communication Systems Engineering, Faculty of \\ Engineering, \\ UPM Serdang \\ ${ }^{2}$ Department of Computer Science, \\ Faculty of Computer Science and Information Technology, UPM Serdang \\ chuiyewleong@hotmail.com,arr@upm.edu.my,thinagaran@upm.edu.my
}

\begin{abstract}
IP based network public address (PA) is an audio management system that provides high flexibility in reconfiguration, system expansion, and requires much simplified cabling structure. This translates to significant cost saving in installation cost, both labour and material, as it requires less cabling, reduced cable size, condoit size, and trunking size. The network PA works very much similarly to computer networks where packets of data are transmitted from one node to another with each of them having a unique IP address in the network system. The nodes will then on Gyick up the packets that solely addressed to them. The network PA can also be integrated with othe building services or subsystems that are TCP/IP compliant such as the five Gldrm management system, CCTV system, and building management system to provide instantaneous volce assisted response and feedback from each system.
\end{abstract}

Keywords: IP, Network Public Address, Intelligent Building

\section{Introduction}

The development of today's industrial electronics industry has an immense impact on the audio technology especially in the public address (PA) system industry. This provides a solution for intelligent building owners and consultants for a more reliable and flexible audio signal management system. These advancements have also resulted in the development of an automated early evacuation solution to any building regardless of its built. An audio matrix system is the solution to the above issue. Generally, a system is classified as a matrix system if it is able to route multiple inputs to multiple outputs simultaneously. This includes simultane us paging to different zones as well as differing background music to various zones. Yet a matrix system would not be physically confined to a particular signal routing pattern and users should be able to change the routing pattern easily via software as and when required [1-3]. However, in recent times, designing and building audio matrix system in conventional approach with certain degree of flexibility and intelligence has been a difficult and an expensive undertaking. This is due to the requirement of large expenditures on wiring conduit, switching equipment and installation labor. In addition, conventional audio matrix systems built with hybrid analog and digital technologies reflects some drawback in system modification and prohibitively expensive to reconfigure once installed. Hence, the network based matrix system seems to have huge potential to providing comprehensive solution over the flaws of the conventional audio matrix system. In next sections, the design and 
development of network PA is illustrated in detail. The outline of the paper is as follow. Section 2 presents the background and related work and Section 3 the system architecture. Section 4 describes the implementation and followed by experimental design and analysis. Conclusions are drawn in Section 5.

\section{Related Works}

In general, the public addressing (PA) system technology has gone through two generations of development phases before reaching to the current network based implementation. The first generation is switcher based PA system, which is capable to carry only two simultaneous broadcasts at the most. These consist of one background music (BGM) source and one paging microphone source. The switcher based PA system has several inherent flaws in limiting the capability and flexibility of audio distribution applications. First, each zone can only be either listening to background music or paging announcement. Second, the switcher based PA system does not allow simultaneous paging and simultaneous background music broadcast. Hence, for a system with two paging,consoles, only one paging console (MIC A or MIC B) can be in use at the same time. Third there is only a single BGM source player can be selected to all zones at any one time. In other words, there is either BGM 1 or BGM 2 can be routed to designated zones at any given time.

The second generation of PA system technology is audio matrix PA system. The audio matrix PA system differs to switcher based PA system by providing simultaneous audio routing of inputs to outputs. This is achieyed by the usage of an audio matrix bus. The audio matrix PA system has several major adyantages over the conventional switcher based PA system. First, each individual zone is capable to listen to the dedicated background music as preferred. Second, the audio matrix PA system allows multiple simultaneous paging and background music broadcast at the same time. For example, four paging consoles are allowed to use simultaneously to make announcements to various zones, as long as there are no conflicts of the zones. Besides, the ardio matrix PA system also allows multiple BGM broadcasts to varying zones. Hence, in application wise, this allows zones to have dedicated BGM broadcast whêre required. Third, the audio matrix PA system is pioneering to introduce software base control and configuration interfaces in order to allow various automated functions to be programmed, such as, automatic time scheduled digital announcements and automated concerted voice evacuation. The latest generation of PA system technology is net or k-based implementation in standard Ethernet infrastructure. The system is designed for garrying audio over conventional Ethernet network (10/100-Base-T), preferably using a dedicated Ethernet or Virtual LAN (VLAN) switched Ethernet for audio routing purposes. In the aspect of PA system industry, there are two major development trends of network-based implementation approach: data link layer, and IP network layer. The data link layen system adopts its own protocol identifier at the data link layer itself. So, it does notuse Internet Protocol (IP) for data routing and Transmission Control Protocol (TCP) fondata ransport, respectively, in order to avoid overhead due to the complex header structure of TCP/IP. However, due to the absent of IP header, it is only allowed to operate over a Local Area Network (LAN), and it is not capable to support routing over the Wide Area Network (WAN) or Internet. CobraNet [4] and EtherSound [5] are categorized in this type of implementation. On the other hand, the IP network layer system adopts standard TCP/IP protocol stack to implement audio routing and data transport over the LAN and WAN. However, as the trade-off of routing capability, it sacrifices certain amount of traffic efficiency due to the TCP/IP header overhead [6, 7]. In general, the technology of Audio over IP (AoIP) and Voice over IP (VoIP) are categorized in this type of implementation. In 
the next section, the system architecture of the network PA design and implementation is described in depth.

\section{System Architecture}

The implementation of IP network layer based PA system is almost identical to the design of standard TCP/IP network, but with some critical techniques in protocol manipulation. In the perspective of PA industry, the operation of single and multi-zones paging from a signal source is crucial in all the aspect of audio distribution technology. Hence, the capability to support unicast, multicast, and broadcast is imperative in order to emulate individual paging, group paging, and all-call, respectively. However, the uncontrolled adoption of multicast and broadcast in a standard TCP/IP network will cause disaster named "broadcast storm" within the LAN. Thus, it is important to restrict the total number of simultaneous input audio channel within a dedicated LAN or bounded in a VLAN. Otherwise, it is liable to cause starvation of the LAN traffic and disables existing network services, such as DNS and DHCP, which are dependency to the broadcast mechanism to function properly. Besides, it is not suitable to emulate group-paging operation with multicast The reason is, in PA audio distribution, it is common for two paging groups comprise an identical zone as shown in Figure 1. In order to perform paging to Group 1 and Group 2 akernatively, address reconfiguration of Zone 3 is imperative for the case. However, the address reconfiguration process is liable to commit the efficiency of paging operation, especially to incurring extra delay and paging latency. In fact, in PA system industry, a paging console is usually required to perform individual and group paging regularly. For this case, it is not a reasonable solution for a paging console to provide two dedicated network interfaces in supporting individual and group paging concurrently. Hence, the broadcast hechanism has more advantages over the multicast in order to implement gioup paging in IP-based network PA system design [8]. The broadcast mechanism should operate associated with some kind of control methods in order to achieve arbitrary selection of targeted paing zones.

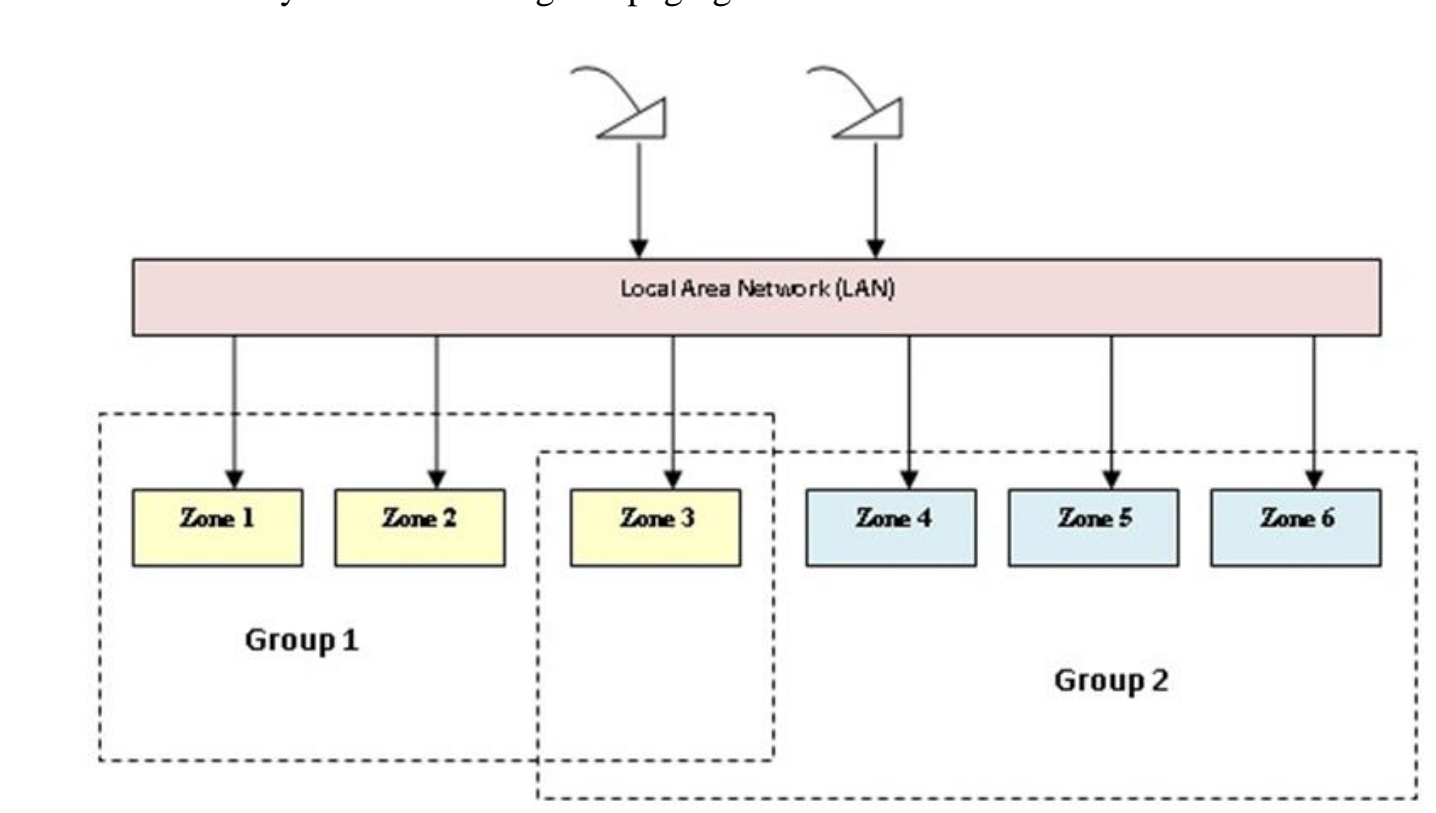

Figure 1. Zone Overlapping of Two Paging Groups 
On the other hand, it is crucial to determine the maximum number of zones within a paging group. For most of the modern PA applications such as Background Music (BGM) audio streaming, it is not an uncommon issue for a paging group to comprise up to hundreds of paging zones. Again, the broadcast mechanism and its counterpart of control methods have shown their capability to accomplish the critical requirement in the most simple and systematic paradigm. In the ideal case of TCP/IP, Class A, B, and C network can support up to 253,65533 , and 16777213 of paging zones, respectively. Besides, multiple paging events are capable to be conducted simultaneously. In other words, a number of simultaneous audio channels are allowed within the Local Area Network (LAN). The performance of the simultaneous audio channels distribution is dependence to the maximum bandwidth of the network port. For 100-Based-T Ethernet, the maximum bandwidth is 100Mbps. The bandwidth consideration is based on the Ethernet switch but not the Ethernet hub, due to the reason of each network port in Ethernet switch has dedicated data path mapping to the others. The corresponding control methods will be discussed in the following section.

\section{A. Implementation of Control Mechanism}

In order to implement control methods of audio distribution within the LAN, it is crucial to clarify the concept of three-step completion. In thi case, a paging event will involve three steps of operations to deliver audio source from a paging console to the desired paging zones. Before starting the discussion about the details of each step, it is important to explain the overall concept of network design in briefly first. In general, each of the network devices must have two ports getting ready for the system, one for control and another one for audio streaming purpose. The control port is always open lo service requestor who intends to occupy the current network device However, the audio streaming port is only open ondemand whenever an authorized requestor is aequiring the service of audio streaming from control port. The control port enables requestor to perform status checking, audio streaming port opening/closing, and priority setting. In other words, requestor is allowed to determine the port number to be opened for audio streaming, as well as to configure the corresponding priority level of the ôpêned port. Based on the concept of control port and audio streaming port, the three-step completion of paging event is defined as a process sequence of corresponding porf adoption in order to accomplish managed audio distribution and switching over the LAN. Figure 2 illus rates the mechanism of three-step completion in flow chart.

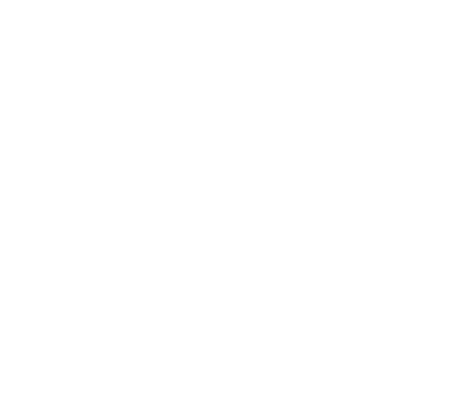




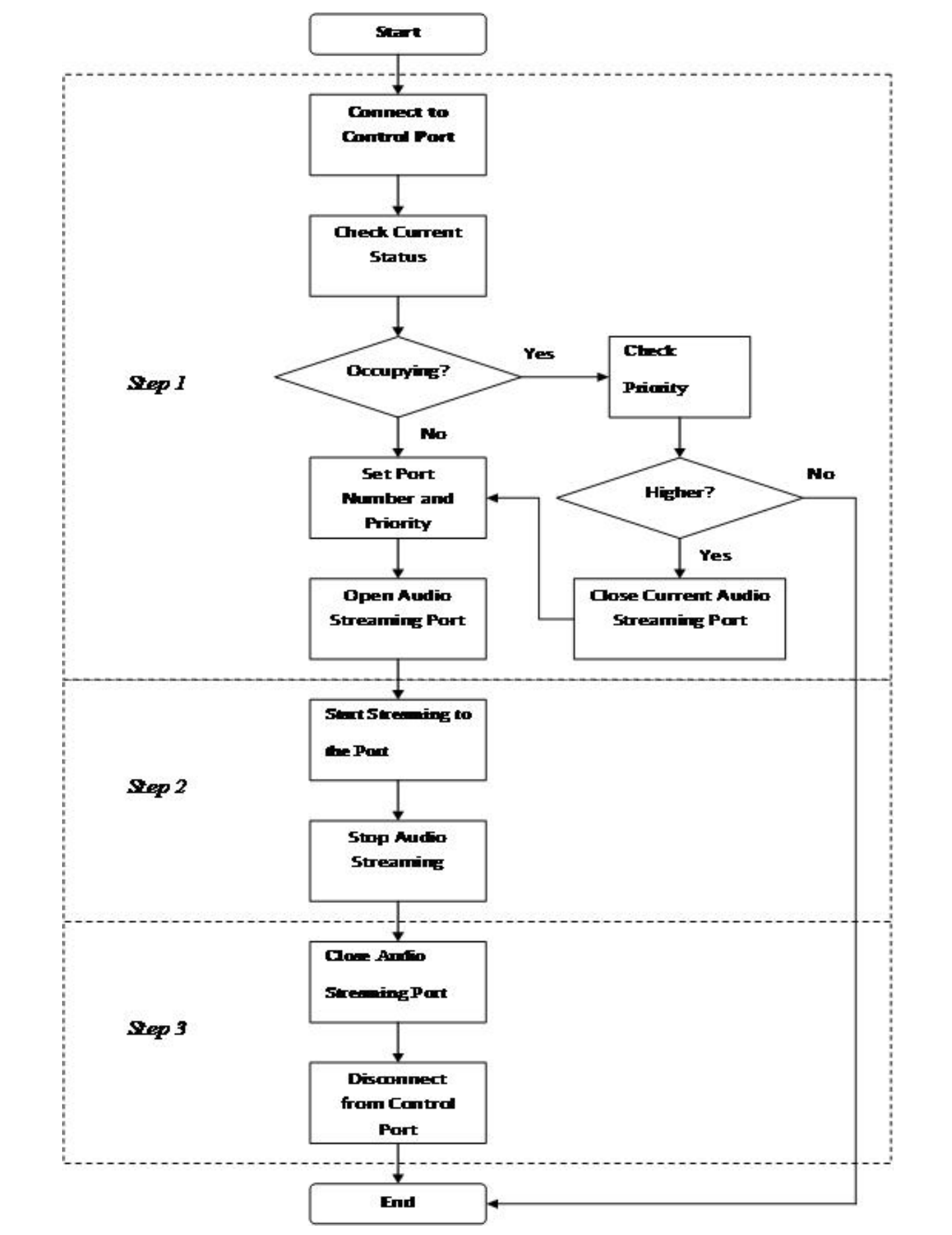

Figure 2. Three-Step Completion of Managed Audio Distribution

Step 1 defines the control mechanism of audio streaming port opening/closing based on the current status feedback from the control port. Once the requestor is connected to the control port, it will request for availability information of the network device. If the network device is not currently occupying by other, the requestor is permitted to set appropriate port number and priority level on the network device. After that, the requestor is allowed to open the 
audio streaming port at the network device. However, if the network device is currently occupying by other, then the requestor will check for the priority level of the audio streaming port that is in service. If the priority level of the requestor is higher than the current occupier in service, the requestor is allowed to close the audio streaming port or performs cut off in runtime. So, the requestor can redefine the port number and priority level on the network device, and open the audio streaming port again. Otherwise, if the priority level of the requestor is lower than the current occupier in service, then the requestor is not allowed to perform cut off as well as open another audio streaming port. In Step 2, the authorized requestor begins to start audio streaming to the corresponding port number with broadcast address. Hence, all the network devices within the LAN are able to receive the audio streaming but only those with appropriate port number opened are enabled to retrieve the audio streaming in order to perform playback at the network device. This method is very efficient to implement group-call as well as all-call in IP-based PA application because each individual audio streaming source is available to be retrieved from all the physical port of network switch within the LAN. The broadcast mechanism is performed and managed by the network switch at data link layer. Thus, for each audio streaming sender, only one audio data packet is required to be sent to the network in order to make available to all the network devices in the LAN. Figure 3 shows the broadcast mechanism of audio streaming over the LAN.

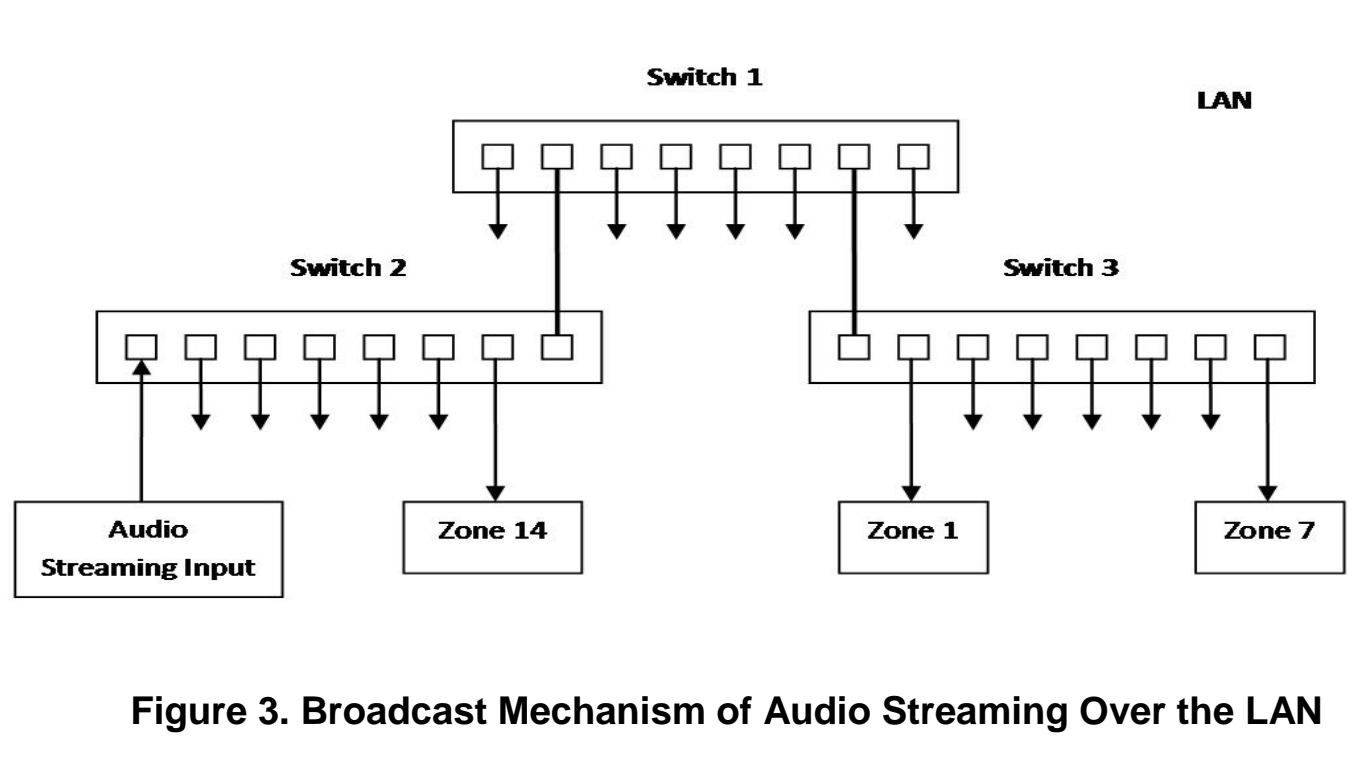

In Figure 3 there are three network switches in the LAN. In the first port of Switch 2, an audio streaming input is delivered into the port as broadcast. So, all the ports in Switch 1, Switch 2, and Switch 3 are available to retrieve the audio streaming source. However, only those ports connected to network devices with identical audio streaming port opened are allowed to perform audio playback at the dedicated zones. Thus, in Figure 3, only Zone 1, Zone 7, and Zone 14 will receive the audio streaming input from Switch 2. On the other hand, for the case of multiple audio streaming inputs, each of the inputs owns a unique port number to be configured as audio streaming port. In other words, each requestor that represents an audio input device will open a dedicated port at the targeted network device for audio streaming purpose. Figure 4 below shows an example that illustrates the operation flow of two requestors to deliver respective audio streaming to two zones individually. 


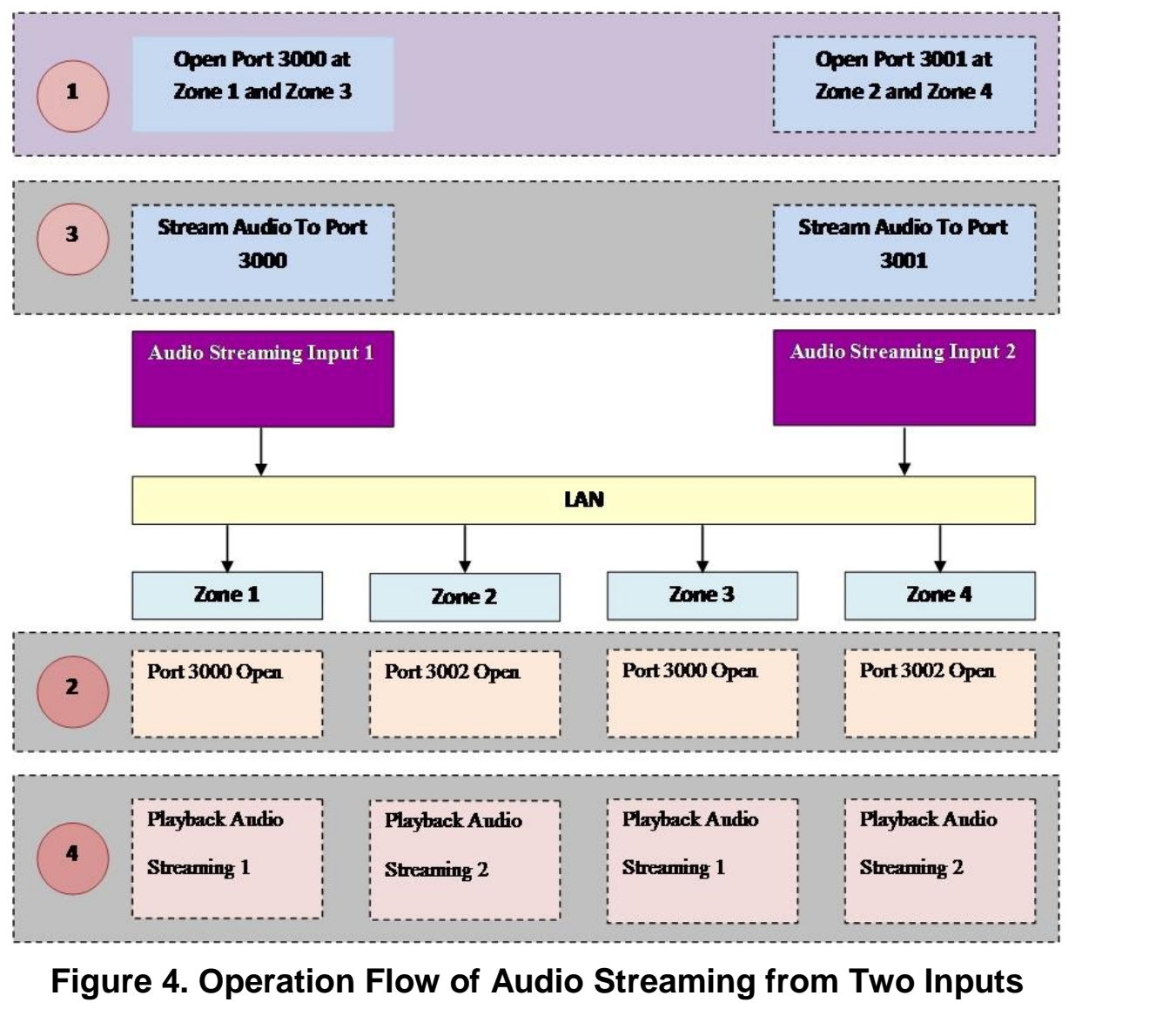

In Figure 4, Audio Streaming Input 1 and 2 are attempting to stream audio to Zone 1, Zone 3 , and Zone 2, Zone 4 respectively. In order to stream to the right zone, each Audio Streaming Input needs to open a port at the desired network device in the corresponding zone accordingly. For the example in Figure 4, Audio Streaming Input 1 needs to open port 3000 at the network devices at Zone 1 and Zone 3 in order to allow these two zones to retrieve its incoming audio stream. After that, the Audio Streaming Input 1 will perform audio broadcast to port 3000 within the AN. Hence, all the zones with port 3000 are opened, which are Zone 1 and Zone 3, Will perform playback of Audio Streaming 1. In Step 3, once the occupier of the dedicated network device is completed to stream audio, it manages to close the audio streaming port and disconnect from the network device. Thus, the network device becomes available to all the requestor again, and ready to process any incoming request for audio streaming purpose. The three-step completion method is very efficient in implementing network system with a lot of output devices. In the case of PA system, each zone will represent by an output device. Hence, for the PA system with 100 output zones, then 100 of net work devices are necessary to support the system. In fact, the design of PA system is very critical to two issues: the maximum number of output zone, and the maximum number of simultaneous audio channel. Regarding to the first issue, the three-step completion method is appropriate because the broadcast mechanism can support up to 254, 65534, and 16777214 of output zones in Class-C, Class-B and Class-A network, respectively. On the other hand, for the case of maximum simultaneous audio channel, it is crucial to emphasise the importance of broadcast mechanism in contributing to the performance of network traffic. In switched network, the switch can recognize the destination address and can route the frame from the 
source port to the destination port to which the destination device is connected, and the rest of the ports are not involved in the transmission process. Besides, the switch can receive another frame from another station at the same time and can route this frame to its own final destination without collision. However, the processes of recognizing destination address and performing port route will commit the resources of switch. Instead, broadcast will bypass the committing processes and route the frame to all the ports directly. Hence, the broadcast mechanism will consume less resources of the switch and provide better transmission performance, which is supported by the test results as shown in the next section.

\section{B. Performance Evaluation}

The broadcast mechanism in three-step completion was tested in a dedicated LAN and the network performance was measured in terms of Round Trip Time (RTT) and Jitter. The measurement process was targeted to study the relationship of network performance level and the number of simultaneous audio channel within the LAN. The number of simultaneous audio channel was measured from 0 with increment of 10 until up to 30 For each measurement of simultaneous audio channel, the RTT and Jitter was sampled in each 10 seconds for 10 minutes. Hence, each measurement of simultaneous audio channel will obtain 60 samples of RTT and Jitter. Figure 5(a) presents the results of RTT versus measurement time (in each 10 seconds) for $0,10,20$, and 30 simultaneous audio channels with broadcast mechanism in line chart.

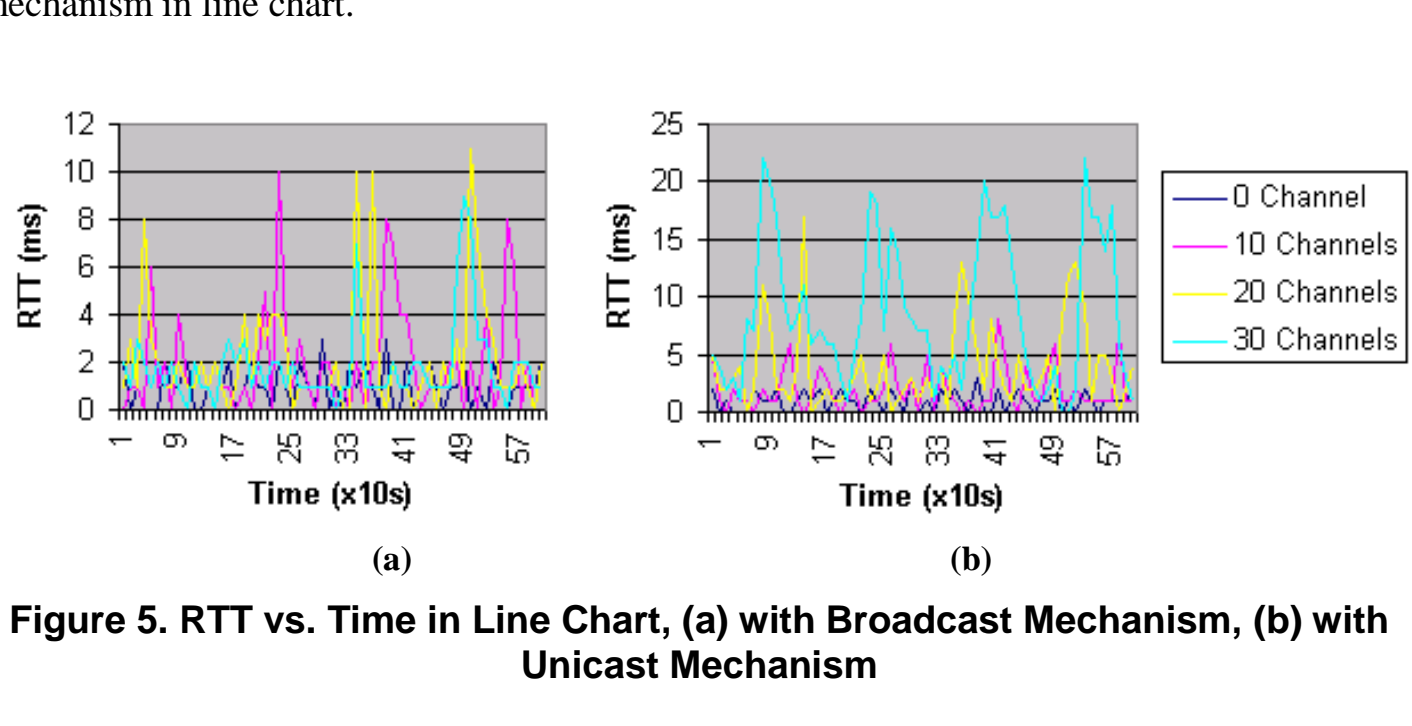

From Figure 5(a), there is obvious different from 0 channel to 10 channels. However, there is no obvious variation from 10 channels to 20 and 30 channels. Besides, the maximum level of RTT is occurred in 20 channels with $11 \mathrm{~ms}$. Since the increment of simultaneous audio channel does not show a proportional relationship with the RTT, then it means the 30 channels is yet to reach a level in differentiating the network capacity over the network performance proportionally. As a comparison to the broadcast mechanism, Figure 5(b) shows the results of RTT versus measurement time with unicast mechanism in line chart. In Figure 5(b), RTT shows significant different over the time for 0 channels to 10 channels, 10 channels to 20 channels, and 20 channels to 30 channels. Hence, the increment of simultaneous audio channel has shown a proportional relationship with the RTT, and the maximum level of RTT is occurred in 30 channels with $22 \mathrm{~ms}$, which is double of the maximum RTT in broadcast mechanism. So, it is evident that the broadcast mechanism has better network performance over the unicast mechanism in processing simultaneous audio channel transmission. On the 
other hand, network jitter is also an important parameter in order to represent the performance of network traffic. Figure 6(a) shows the results of jitter versus measurement time (in each 10 seconds) for $0,10,20$, and 30 simultaneous audio channels with broadcast mechanism in line chart.
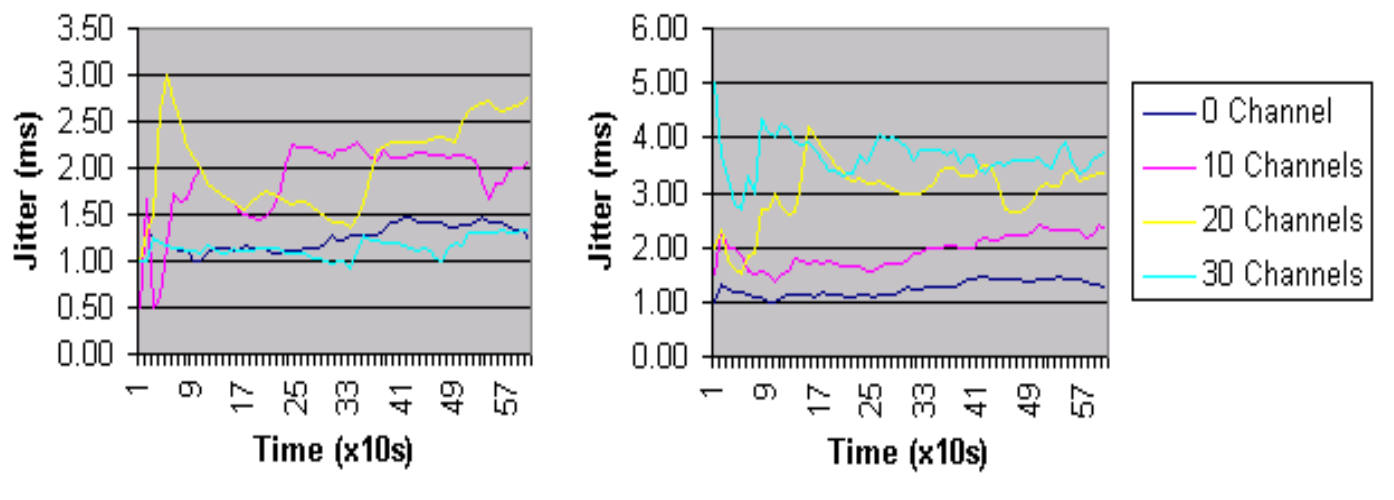

(a)
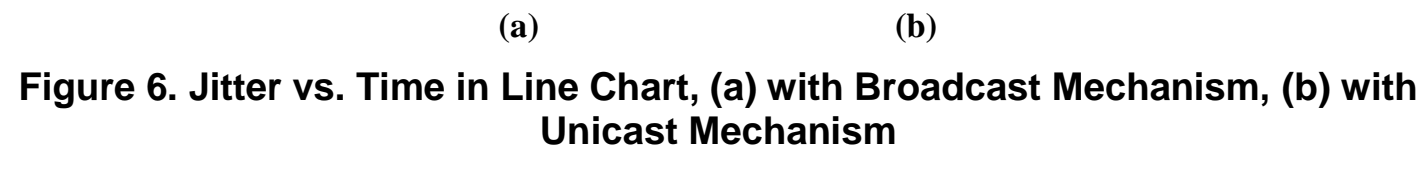

Again, based on the results from Figure 6(a), the obvious different is only existed from 0 channels to 10 channels and there is nocobvious variation from 10 channels to 20 and 30 channels. Besides, the maximum level of jitter is oocurred in 20 channels with $3.00 \mathrm{~ms}$. So, the increment of simultaneous audiochannel also does not show a proportional relationship with the jitter, and again it means the 30 channels is yet to reach a level in differentiating the network capacity over the network performance proportionally. As a comparison to the broadcast mechanism, Figure 6(b) shows the results of jitter versus measurement time with unicast mechanism in line chart. In Figure 6(b), jitter shows significant different over the time for 0 channels to 10 channels, 10 channels to 20 channels, and 20 channels to 30 channels. Hence, the increment of simultaneous audio channel has shown a proportional relationship with the jitter, and the maximum level of jitter is occurred in 30 channels with $5 \mathrm{~ms}$. As comparison, the max mum level of jitter in broadcast mechanism is only $3 \mathrm{~ms}$. Thus, based on the experimental results of both RTT and jitter, it is undoubtedly to show that the broadcast mechanism has better network performance over the unicast mechanism in implementing simaltaneous audio transmission over the network for IP based public address system design,

\section{Conclusions and Future Work}

In this paper, the mechanism of IP based public address has been shown experimentally based on the audio streaming implementation over network. Hence, the adoption of broadcast mechanism in three-step completion process is reasonable in order to deliver high performance managed audio distribution over the LAN infrastructure. This is suitable to be applied in IP based network public address system, which reflects critical concern on the capacity of simultaneous audio channels within the network. As for future works, the proposed method in managed audio distribution will be expanded to include image and video transfer over the LAN. 


\section{References}

[1] AEX System, "Public Address System Products", http://www.aexsystem.com, Accessed on Nov 2010.

[2] Bosch, "Public Address (PA) and Voice Alarm",http://www.boschsecurity.com, Accessed on Nov 2010.

[3] TOA Corporation, "PA (Public Address) \& Sound Equipment", http://www.toa.jp, Access on Nov 2010.

[4] CobraNet, "CobraNet Networked Digital Audio",http://www.cobranet.info, Accessed on Nov 2010.

[5] EtherSound, "EtherSound Technology",http://www.digigram.com, Accessed on Nov 2010.

[6] Nilsson M.E. et al., "Adaptive Multimedia Streaming Over IP”, IEEE International Conference on Visual Information Engineering, 2003.

[7] H. Akagi, "IP-based AV Home Network System", IEEE Consumer Communications and Networking Conference, (2005).

[8] R. Yin, "Medium Access Control with Packet Length Priority towards a Real- Time Ethernet, America Control Conference, (2005).

[9] S. T. Bushby, "Control Systems Research for Building Automation, American Control Conference, (2007), ACC '07, 2007, pp. 7-8

[10] J. D. Day and H. Zimmermann, "The OSI reference model", Proceedings of the IEEE, vol. 1 pp. 13341340, (1983).

[11] M. Song, K. Ohkyn and C. Yunmo, "A serial connection technique of speakers for multi-channel audio systems", IEEE Transactions on Consumer Electronics, vol. 51, (2005), pp. 611-616.

[12] S. G. Servent, M. Duzynski, M. Scholles, P. Nauber and U. Schelinski, Cycle time ynchronization among IEEE 1394 clusters via UWB over coaxial cable for HANA and AV/C consumer digital multimedia networks," IEEE Transactions on Consumer Electronics, vol. 55, (2009), pp. 535-540.

\section{Authors}

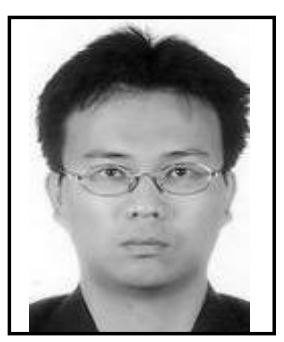

Chui Yew Leong received his B.Eng. Electronics in 2000 from Universiti Malaysia Sabah and M.Sc. specializing in Computer Engineering in 2003 from Universiti Putra Malaysia He obtained his $\mathrm{Ph} D$ drom same university. His main interests are embedded system design, network security and intelligent systems. Currently he is attached as postdoctoral fellow with Institute of Advanced Technology, Universiti Putra Malaysia. He is a member of IEEE

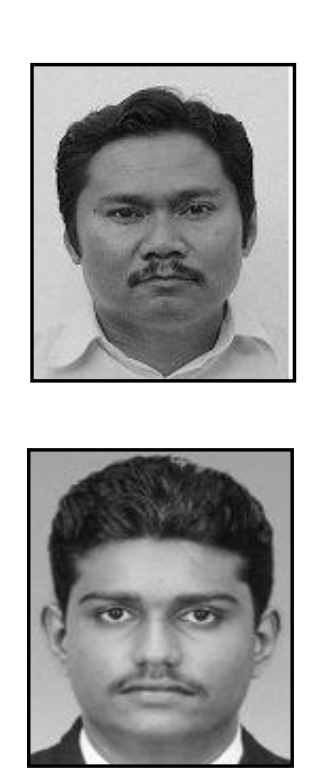

AbdRahman Ramli received M.Sc. degree in Information Technology System from University of Strathclyde, United Kingdom in 1985 and Ph.D. in Image Processing from University of Bradford, United Kingdom in 1995. His main interests are imaging, image processing systems and intelligent systems. He is a member of IEEE.

Thinagaran Perumal received his B.Eng. in 2003 at Department of Computer and Communication Systems Engineering, Universiti Putra Malaysia. He completed his M.Sc. degree in Intelligent Systems and Ph.D. in Smart Technology and Robotics at Institute of Advanced Technology, Universiti Putra Malaysia. His main interests are smart home systems, middleware technologies and embedded system design. He is a senior member of IEEE. 